\title{
Medical Council of Canada abandons controversial exam
}

Cite as: CMAJ 2021 July 5;193:E1014-5. doi: 10.1503/cmaj.1095947

Posted on cmajnews.com on June 10, 2021

T he Medical Council of Canada (MCC) has scrapped a controversial medical licensing exam after repeated cancellations and technical difficulties during the pandemic.

In a June 10 statement, the organization confirmed it is "stepping away" from the MCC Qualifying Examination (MCCQE) Part II, citing the impact of the pandemic and the unprecedented challenges associated with delivering the test.

Going forward, the MCC will grant a Licentiate of the Medical Council of Canada (LMCC) - a key hurdle on the path to full medical licensure - to anyone who

- Graduated from an acceptable Canadian or international medical school or American school of osteopathic medicine;

- Successfully completed the MCCQE Part I;

- Successfully completed at least 12 months of acceptable postgraduate medical or osteopathic clinical training; and

- Has the required medical credentials, including verification of postgraduate training or evidence deemed acceptable by the MCC's executive director.

On May 31, the organization suspended the virtual delivery of the MCCQE Part II in the middle of spring sittings after technical difficulties prevented at least half of the candidates on one day from completing the test. Last year, the MCC cancelled in-person sittings in the spring and fall on short notice due to pandemic concerns.

The MCC says it will refund exam fees to candidates registered for the latest session within the next 30 days, but it may take up to two months to award them certification under the new rules.

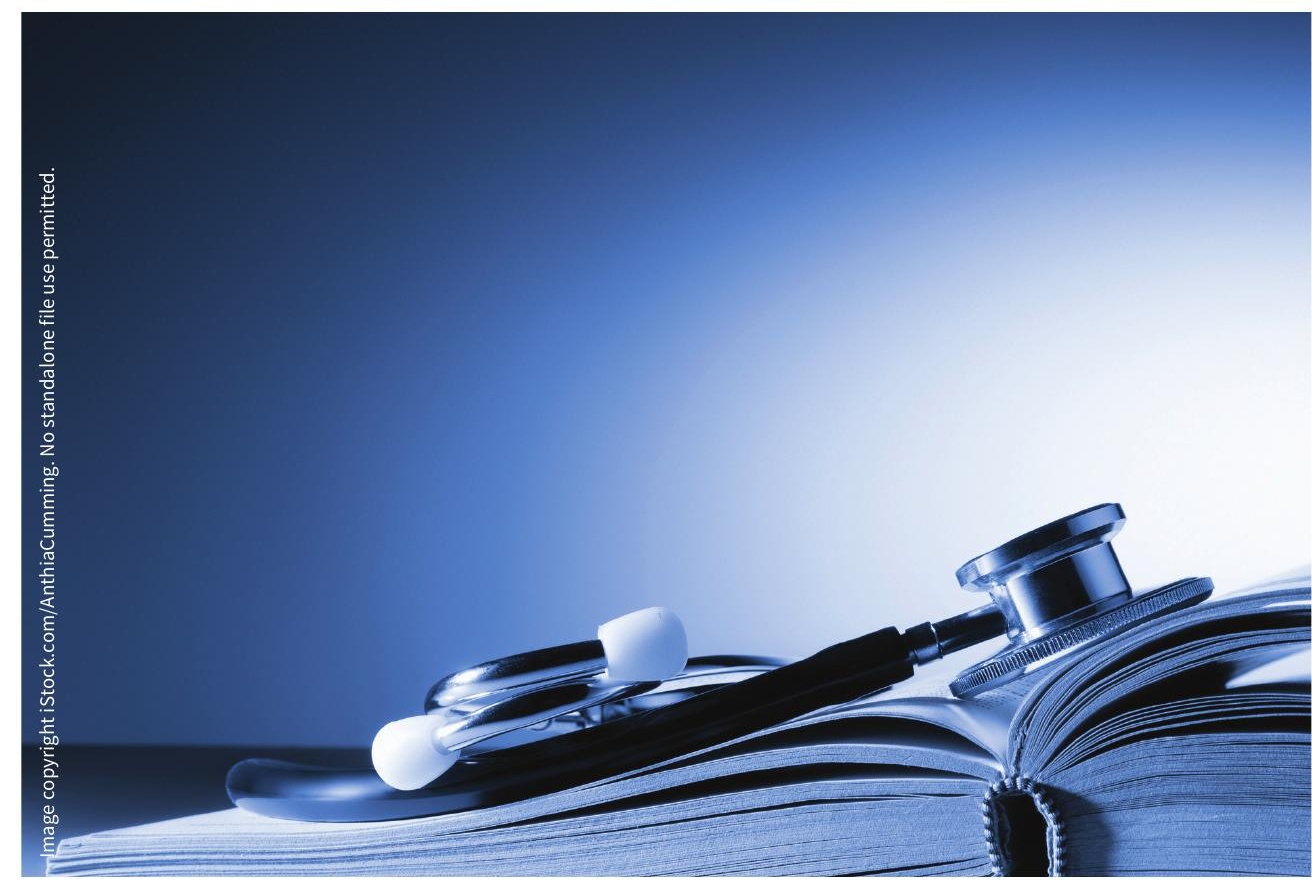

Resident doctors will no longer be required to pass the MCCQE II as part of their journey to independent practice.

For other eligible candidates, the process will require coordination with third parties and may take longer.

Many resident doctors have prepared for the exam multiple times over the past year and have lost time and money due to the last-minute cancellations. Meanwhile, their careers have hung in limbo.

"This was my third time preparing for this exam," one resident told CMAJ. "Most days I spend more than 15 hours at work and studying without a real break." She described feeling burned out and stressed from missing learning opportunities, vacation time, and time with her family because of the exam.

Several other residents wrote to CMAJ with similar stories. They described spending hours preparing and travelling to write the test, taking unpaid leave, and missing clinical experiences and career opportunities - only to have their exams terminated with little warning or explanation, in some cases during their sittings.

"We are so tired and so done," said one resident whose latest exam was cancelled with 48 hours' notice. "The MCC has shown a complete lack of professionalism, an inability to competently conduct an exam and behaviour frankly unbecoming of the medical profession."

A spokesperson for the MCC told CMAJ that the organization regrets the challenges faced by exam candidates. "We certainly take responsibility and empathize with the stress the entire disruption of the pandemic has caused 
in personal and professional lives, including exam disruptions. It was certainly not our intended outcome."

Resident doctors have questioned the relevance of the MCCQE Part II in recent years, especially as some have been practising under provisional licenses since the spring without completing the exam.

The MCC has maintained the importance of standardized testing of generalist competencies like assessment, diagnosis, and professional behaviour.

It also appears the organization is leaving the door open to reintroduce the exam or something like it down the road. According to the MCC, "criteria for the award of the LMCC may be reviewed at a future date as standardized assessment requirements for physicians evolve."

However, critics of the MCCQE Part II have pointed out that the same competencies are now evaluated continuously throughout medical training, rendering the exam obsolete.
"I am glad they are finally making a common-sense decision, but this statement still leaves a lot unexplained," said Dr. Michelle Cohen, a rural family physician who has advocated for the exam to be scrapped. "We don't know how this will affect international medical graduates. We don't know if the MCC will try to create another exam and what that might mean for certification. And I'm still hearing anecdotal reports of residents having trouble getting refunds and standardized patients not getting paid for their work - which the MCC so far has not publicly addressed."

At least one actor involved in simulating patient interactions during the latest exams described "chaos" behind the scenes, noting that some actors were asked to grade exam candidates without clinical examiners present. As of June 9, he said it was unclear when the MCC would pay the actors for the days they had worked.
Asked about the payment of standardized patients, the MCC told CMAJ on June 11 "we are still looking into this and working with the [examination] sites."

The organization also confirmed that the trained actors may "score some aspects of a candidate's performance," depending on their role.

As for international medical graduates, those who meet the new criteria "will be granted the LMCC." According to a spokesperson, "at this time, it is not expected the MCCQE Part II will be reintroduced."

\section{Lauren Vogel, CMAJ}

Content licence: This is an Open Access article distributed in accordance with the terms of the Creative Commons Attribution (CC BY-NC-ND 4.0) licence, which permits use, distribution and reproduction in any medium, provided that the original publication is properly cited, the use is noncommercial (i.e., research or educational use), and no modifications or adaptations are made. See: https://creativecommons.org/ licenses/by-nc-nd/4.0/ 